\title{
A Rare Complication of Intravesical Bacillus Calmette Guérin Therapy: Local Progressive Pelvic Inflammation
}

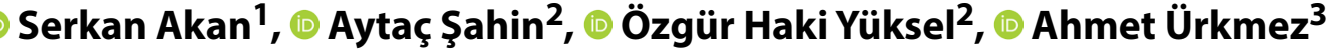

${ }^{1}$ Department of Urology, Health Science University, Sultan Abdulhamid Han Training and Research Hospital, Istanbul, Turkey ${ }^{2}$ Department of Urology, Health Science University, Fatih Sultan Mehmet Training and Research Hospital, Istanbul, Turkey

${ }^{3}$ Department of Urology, Health Science University, Haydarpasa Numune Training and Research Hospital, Istanbul, Turkey

\begin{abstract}
It has been proven that intravesical Bacillus Calmette-Guérin (BCG) therapy prevents the recurrence and progression of carcinoma in situ and of superficial bladder cancers, although local and systemic complications have been reported. However, careful attention should be given to the timing and management of this therapy because complications that may delay possible definitive treatment may also be seen. A case characterized with extensive inflammation, observed rarely after BCG therapy, involving pelvic organs and leads up to the internal abdomen and mesenteric area in the superior and levator muscle and prepubic area in inferior is presented here.

Keywords: Bacillus Calmette-Guérin; bladder cancer; pelvic inflammation.
\end{abstract}

$I^{t}$ t has been proven that intravesical Bacillus CalmetteGuérin (BCG) therapy prevents the recurrence and progression of carcinoma in situ and of superficial bladder cancer. However, it has been associated with local and systemic side effects ${ }^{[1]}$. A case characterized with extensive inflammation observed rarely after BCG therapy and involving pelvic organs and leads up to the internal abdomen and mesenteric area in the superior and levator muscle and prepubic area in the inferior is presented here.

\section{Case Report}

A 59-year-old male patient had transurethral bladder tumor resection for bladder cancer diagnosed as a 4-cm mass on urinary ultrasonography at an external center. Without a second-look transurethral resection, BCG therapy was initiated with T1 high-grade (HG) pathological stage. A 6-week induction treatment was completed. Then, the patient was referred to our clinic. On evaluation, there were no additional comorbidities or other surgical procedures. On physical examination, inflammation and apparent hardness with palpation, including bilateral scrotum and testicles and reaching up to both the inguinal areas and prepubic area, were noted. On anamnesis, it was observed that these conditions persisted even before the last induction cure. Next, empirical quinolone treatment was initiated, and additional radiological and laboratory examinations and consultation to pulmonary and infection disease clinics were

Correspondence (iletişim): Ahmet Urkmez, M.D. Department of Urology, Health Science University, Haydarpasa Numune Training and Research Hospital, Istanbul, Turkey

Phone (Telefon): +90 5077668300 E-mail (E-posta): ahmeturkmez@hotmail.com

Submitted Date (Başvuru Tarihi): 21.02.2018 Accepted Date (Kabul Tarihi): 08.03.2018

Copyright 2018 Haydarpaşa Numune Medical Journal

This is an open access article under the CC BY-NC license (http://creativecommons.org/licenses/by-nc/4.0/). 
requested. When the pathological samples were re-evaluated at our hospital, the pathological stage observed was T2HG. There was no pulmonary involvement. On radiological evaluation, an extensive, local, progressive, nontumoral inflammation involving the pelvic organs and leading up to the mesenteric area in the abdomen in the superior and levator muscle and prepubic area in the inferior was detected (Figs. 1, 2). A 6-month antituberculosis treatment (oral rifampin, isoniazid, and ethambutol) was planned for the patient and because of this condition, definitive treatment was deferred.

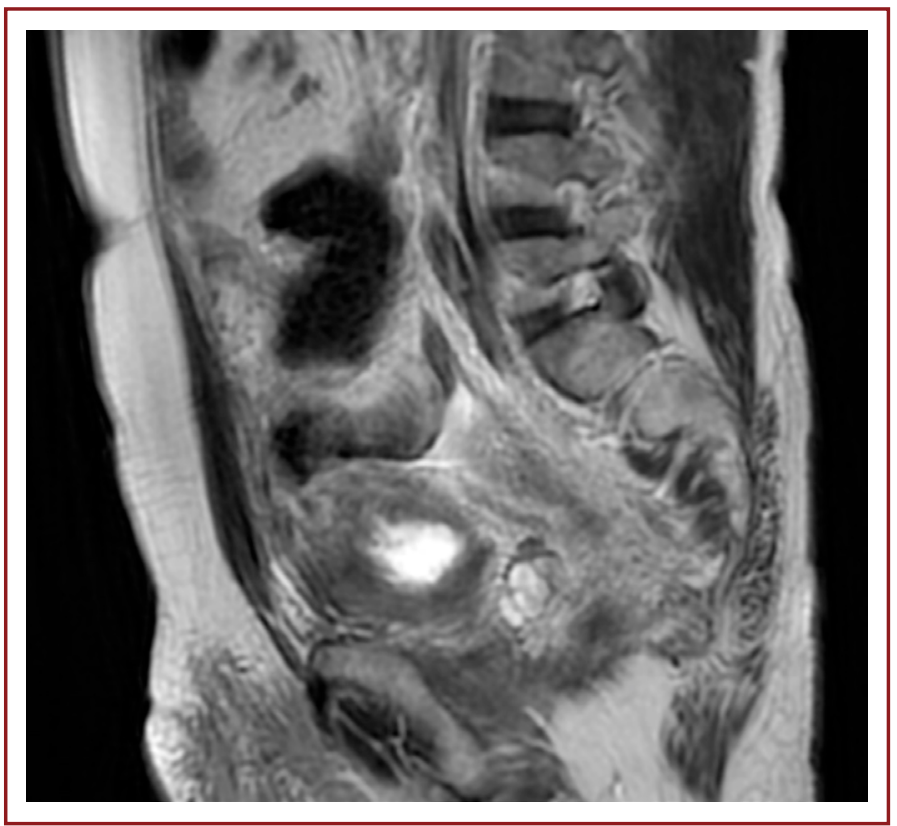

Figure 1. Widespread inflammation extending to the prepubic and perirectal area and left levator muscle.

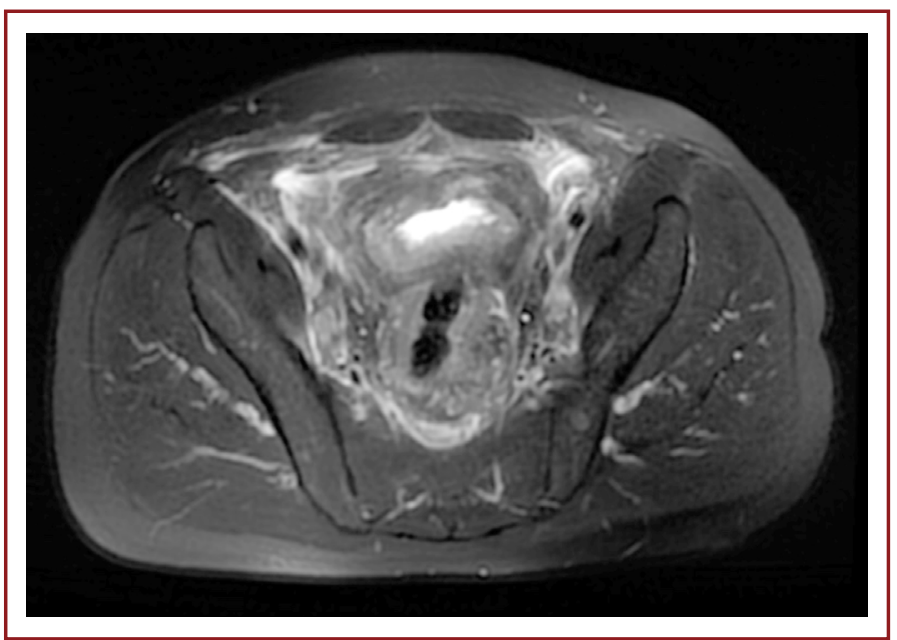

Figure 2. Diffuse thickening of the bladder wall, especially at the right anterolateral wall. This thickening on the right anterolateral wall keeps the entire wall and infiltrates the perivesical fatty planes.

\section{Discussion}

BCG therapy, which is frequently used in medium- and high-risk superficial bladder cancer and carcinoma, decreases their recurrence rates and delays progression ${ }^{[2]}$. It exhibits these effects through the development of T-cellmediated immune response in the bladder ${ }^{[3]}$. It causes local inflammation after instillation and tumor cell damage.

Local complications, such as hematuria, dysuria, and cystitis, are common after BCG application. In the literature, the reported incidence is $57 \%-91 \%$ for hematuria, $26 \%-55 \%$ for cystitis, and $28 \%-73 \%$ for fever ${ }^{[4]}$. Nonsteroidal anti-inflammatory, antipyretic, and antispasmodic drugs may be commonly used to treat spontaneously recovering local complications with a mild course.

Post-BCG systemic complications have been reported but are relatively rare. Systemic complications occur with hematogenous spreading of the bacteria due to predisposing conditions, such as damaged and/or inflammed urothelium caused by extreme tumor resection, diabetes, immunosuppression, liver function disorder, traumatic catheterization, and bladder perforation ${ }^{[5]}$. General condition disorder, myalgia, arthralgia, headache, fever, pneumonia, abscess, and sepsis may also be obserevd. Complications, such as psoas abscess, septic shock, peritonitis, disseminated intravascular coagulation, and diffused granulomatous mesenteric disease have also been reported ${ }^{[6,7]}$.

Aseptic conditions should be maintained during BCG application to prevent the occurrence of secondary complications, and the application should be delayed in case of inflammatory disease, macroscopic hematuria, and cystitis. BCG should be applied at least 2 weeks after transurethral operation and traumatic catheterization. BCG should not also be applied in case of immunosuppression, pregnancy, or previous hypersensitivity. While some researchers recommend isoniazide prophylaxis to prevent these complications, others indicate that this application decreases the antitumoral effect of BCG or fails to prevent these complications ${ }^{[8]}$. Low-dose BCG application has been attempted by many researchers with the same concern, but the results remain controversial.

BCG instillation should be immediately cut when systemic complications occur, and patients with high fever should be immediately hospitalized [9]. Systemic BCG infection treatment is a 6-month regimen, including isoniazide (300 $\mathrm{mg} / 24 \mathrm{~h}$ ), rifampicin ( $600 \mathrm{mg} / 24 \mathrm{~h}$ ), and ethambutol (1200 $\mathrm{mg} / 24 \mathrm{~h}$ ) [3]. Although the use of corticosteroids is risky during treatment, they may be administered in case of high fever, sudden hypotension, and hypersensitivity. 
It is proclaimed that a single transurethral resection (TUR) may not be enough to extract the ill tissue, especially in multiple and invasive tumors, and may cause early recurrence and stage advancement despite additional intravesical treatment ${ }^{[10]}$. Another fact that should not be ignored is that intravesical treatment provided in addition to TUR does not compensate the inadequate resection in TUR [11]. Therefore, second TUR (re-TUR) should ne applied after 2-6 weeks in these patients. In the literature, residual tumor detection ratios in re-TUR range from $28 \%$ to $74 \%$ and understating ratios from $1.7 \%$ to $64 \%{ }^{[12,13]}$. Schwaibold et al. ${ }^{[14]}$ evaluated 136 patients with $\mathrm{T} 1( \pm \mathrm{CIS})$ tumor who had reTUR and observed upstaging in re-TUR in 28 patients (21\%) whose first resection was reported to be standard TUR ${ }^{[15]}$. In the same study, it was found that re-TUR applied before BCG prevented upstaging with an additional ratio of $27 \%$ compared with BCG alone. Thus, re-TUR is recommended in multifocal disease, large tumor $(\geq 3 \mathrm{~cm})$, incomplete resection, and T1HG pathological stage.

In our case, the pathological stage for standard TUR operation applied in an external center was evaluated as T1HG, and BCG treatment was applied because re-TUR was regarded unnecessary due to complete resection. The patient was referred to our center due to the development of progressive pelvic inflammation because of BCG. Muscular invasion was observed on histopathological re-evaluation by an experienced uropathologist at our center. Although we believed that cystectomy should be immediately performed in this young high-risk patient without long-term metastasis, the definitive treatment decision had to be delayed due to BCG-related intense pelvic and mesenteric inflammation.

\section{Conclusion}

Although BCG therapy has a rare local and systemic side effect, it is an effective treatment for carcinoma in situ and superficial bladder cancer. However, complications that may delay possible definitive treatment, as in this case, may also be observed. Therefore, particularly regarding re-TUR indication, we believe that it should be performed in pathologically and radiologically noninvasive patients, and this will not result in delay in the possible definitive treatment.

Informed Consent: Written informed consent was obtained from the patient for the publication of the case report and the accompanying images.

Peer-review: Externally peer-reviewed.

Conflict of Interest: None declared.
Authorship Contributions: Concept: S.A.; Design: O.H.Y.; Data Collection or Processing: S.A.; Literature Search: S.A., A.S.; Writing: S.A., A.S., O.H.Y., A.U.

Financial Disclosure: The authors declared that this study received no financial support.

\section{References}

1. Rischmann P, Desgrandchamps F, Malavaud B, Chopin DK. BCG intravesical instillations: recommendations for side-effects management. Eur Urol 2000;37:33-6. [CrossRef]

2. Kurth $\mathrm{KH}$, Bouffioux C, Sylvester R, van der Meijden AP, Oosterlinck W, Brausi M. Treatment of superficial bladder tumors: Achievements and needs. The EORTC Genitourinary Group. Eur Urol 2000;3:1-9. [CrossRef]

3. Soylu A, Ince AT, Polat H, Yasar N, Ciltas A, Ozkara S, et al. Peritoneal tuberculosis and granulomatous hepatitis secondary to treatment of bladder cancer with Bacillus Calmette-Guérin. Ann Clin Microbiol Antimicrob 2009;8:12. [CrossRef]

4. Suzuki S, Shinohara N, Harabayashi T, Taniguchi A, Haga K, Sato $S$, et al. Complications of bacillus Calmette-Guerin therapy in superficial urothelial cancer: clinical analysis and implications. Int J Clin Oncol 2002;5:289-93.

5. Durek C, Richter E, Basteck A, Rüsch-Gerdes S, Gerdes J, Jocham $D$, et al. The fate of bacillus Calmette-Guerin after intravesical instillation. J Urol 2001;165:1765-8. [CrossRef]

6. Alvarez-Múgica M, Gómez JM, Vázquez VB, Monzón AJ, Rodríguez JM, Robles LR. Pancreatic and psoas abscesses as a late complication of intravesical administration of bacillus Calmette-Guerin for bladder cancer: a case report and review of the literature. J Med Case Reports 2009;3:7323. [CrossRef]

7. Shapiro A, Vakar F, Kamat AM. Diffuse granulomatous mesenteric disease caused by intravesical bacillus Calmette-Guérin instillation masquerading as peritoneal carcinomatosis. Urol Oncol 2005;23:352-3. [CrossRef]

8. van der Meijden AP, Brausi M, Zambon V, Kirkels W, de Balincourt C, Sylvester R; Members of the EORTC Genito-Urinary Group. Intravesical instillation of epirubicin, bacillus Calmette-Guerin and bacillus Calmette-Guerin plus isoniazid for intermediate and high risk $\mathrm{Ta}, \mathrm{T} 1$ papillary carcinoma of the bladder: a European Organization for Research and Treatment of Cancer genito-urinary group randomized phase III trial. J Urol 2001;166:476-81. [CrossRef]

9. Koga H, Kuroda M, Kudo S, Yamaguchi A, Usami M, Suzuki T, et al. Adverse drug reactions of intravesical bacillus Calmette-Guerin instillation and risk factors of the development of adverse drug reactions in superficial cancer and carcinoma in situ of the bladder. Int J Urol 2005;12:145-51. [CrossRef]

10. Herr HW, Donat SM. A re-staging transurethral resection predicts early progression of superficial bladder cancer. BJU Int 2006;97:1194-8. [CrossRef]

11. Divrik RT, Yildirim U, Zorlu F, Ozen $H$. The effect of repeat transurethral resection on recurrence and progression rates in patients with $\mathrm{T} 1$ tumors of the bladder who received intravesical mitomycin: a prospective, randomized clinical trial. J Urol 
2006;175:1641-4. [CrossRef]

12. Zurkirchen MA, Sulser T, Gaspert A, Hauri D. Second transurethral resection of superficial transitional cell carcinoma of the bladder: a must even for experienced urologists. Urol Int 2004;72:99-102. [CrossRef]

13. Rigaud J, Karam G, Braud G, Glemain P, Buzelin JM, Bouchot O. T1 bladder tumors: value of a second endoscopic resection
[Article in French]. Prog Urol 2002;12:27-30.

14. Schwaibold HE, Sivalingam S, May F, Hartung R. The value of a second transurethral resection for T1 bladder cancer. BJU Int 2006;97:1199-201. [CrossRef]

15. Herr HW. Restaging transurethral resection of high risk superficial bladder cancer improves the initial response to bacillus Calmette-Guerin therapy. J Urol 2005;174:2134-7. [CrossRef] 